\title{
Visual reweighting in postural control is less adaptative in older adults
}

\author{
José Angelo Barela ${ }^{1,2^{*}}$, Giovanna Gracioli Genoves ${ }^{2}$, Bruno Alleoni ${ }^{2}$, Ana Maria Forti Barela ${ }^{1}$ \\ ${ }^{1}$ Graduate Program in Human Movement Sciences, Institute of Physical Activity and Sport Sciences, Cruzeiro do Sul University, São \\ Paulo, Brazil; ${ }^{*}$ Corresponding Author: jose.barela@cruzeirodosul.edu.br, jbarela@rc.unesp.br \\ ${ }^{2}$ Department of Physical Education, Institute of Biosciences, São Paulo State University, Rio Claro, Brazil
}

Received 27 September 2013; revised 28 October 2013; accepted 12 November 2013

Copyright (C) 2013 José Angelo Barela et al. This is an open access article distributed under the Creative Commons Attribution License, which permits unrestricted use, distribution, and reproduction in any medium, provided the original work is properly cited.

\section{ABSTRACT}

Postural control is based upon the fusion of sensory cues coming from multiple sources requiring continuously adaptation that may be altered due to aging, leading to the poor postural equilibrium in older adults. Therefore, the purpose of this study was to examine the adaptation in the relationship between the visual information and the body sway in older adults. Fifteen older (70 \pm 7.6 years) and 15 younger adults (19 \pm 1.1 years) stood upright inside of a moving room. Each participant performed 7 trials, each lasting $60 \mathrm{~s}$, in which in the first 3 trials the room oscillated at $0.2 \mathrm{~Hz}$, amplitude of 0.6 $\mathrm{cm}$, and peak-to-peak velocity of $0.6 \mathrm{~cm} / \mathrm{s}$. In the fourth trial, the room oscillated at $0.2 \mathrm{~Hz}$ but with amplitude of $3.5 \mathrm{~cm}$ and peak-to-peak velocity of $3.5 \mathrm{~cm} / \mathrm{s}$. In the following 3 trials, the room oscillated with the same parameters of the first three trials. Body sway magnitude was examined through mean sway amplitude, and the relationship between visual information and induced body sway was examined through coherence and gain. Visual manipulation induced corresponding body sway in both older and younger adults, with no difference being observed between groups in the first three trials. In the fourth trial, mean sway amplitude, coherence and gain values were higher for the older compared to younger adults. Moreover, in the last three trials, older adults still showed higher gain values than observed for the younger adults. Taken together, these results suggest that older adults adapt to abrupt changes in visual cues, but not with the same magnitude as younger adults. Yet, older adults do not take advantage of experienced sensory changes in order to adapt the use of the vision information in the following experiences, indicating the less capability of adaptation to the sensory changes.

Keywords: Elderly; Moving Room; Adaptation; Posture

\section{INTRODUCTION}

The maintenance of the upright stance such as during daily activities is characterized by stability and flexibility, in order to accommodate all the changes in the surrounding environment. Therefore, the central nervous system must process a stimulus-rich and continuously changing environment, requiring the unceasing integration of multisensory information to update our estimation of self-motion. Such a mechanism has been denominated as sensory reweighting, which refers to the ability to select and decrease/increase the influence of a specific sensory stimulus on postural control [1].

The concept of sensory reweighting was first introduced over thirty years ago [e.g., 2,3]. More recently, sensory reweighting has been rigorously demonstrated by numerous investigators under conditions in which the amplitude of the sensory stimulus is manipulated with corresponding changes in postural responses, interpreted to reflect a change in weighting to the sensory stimulus in adults [4-8] and even in children [9]. Moreover, these experimental effects have been modeled to understand the mechanistic underpinnings of sensory reweighting within the postural control scheme [4,7,10-12].

Aging is characterized by many changes in several systems, including a decline in postural control performance as evidenced by larger sway magnitude during upright stance $[13,14]$ and the increase of high risk for falling [for review, 15]. This decline has been suggested to occur due to sensory and motor declines [e.g., 15] and how sensory information integration takes place in the 
central nervous system [16,17].

Despite all the efforts in uncovering declines in postural control functioning due to aging and the suggestion that the ability to reweight sensory inputs adaptively is one of the most critical factors for postural control in older adults [16], only recently a more robust approach has been used to investigate sensory reweighting in this population [18]. For instance, Jeka and colleagues [18,19] observed that older adults were more influenced by visual manipulation compared to younger adults. This finding is consistent with previous results $[17,20]$, and indicates that older adults can adapt to abrupt changes in visual manipulation as well as younger adults. However, older adults do not reduce the influence of visual cue throughout the sensory exposition as one can observe in younger adults.

Based upon these evidences, older adults can suppress sensory influences that may threaten postural stability, but such suppression might not be as efficient as in younger adults. Evidence for such suggestion has been observed in previous studies that revealed that older adults couple more strongly to sensory manipulation compared to younger adults $[14,17,20]$. Similar results were observed when the visual information was suddenly removed and re-inserted (closing and opening eyes, respectively) and interpreted as an indicative of less efficient integrative mechanism [21].

In the present study, we employed a strategy to examine the magnitude of sway response to visual manipulation, with different characteristics in several expositions. Considering that sensory integration in older adults seems to be related to many of the postural control changes and that activity-based interventions can improve balance [22], an understanding of the mechanism through which interventions seem to minimize postural control decline must be elucidated in order to target optimal intervention strategies [23]. Moreover, employing a controlled stimulus approach and observing the magnitude of the sway response in older adults in sequential trials may help us to uncover how the central nervous system reconfigures internal postural setting due to the expositions of sensory cues. Therefore, the aim of this study was to examine the adaptation in the coupling between visual information and body sway of older adults due to abrupt visual changes throughout different visual expositions. Our hypothesis was that older adults would couple to visual stimulus as well as younger adults but they would be more influenced by larger stimulus and could not carry over previous experience to the following expositions.

\section{METHODS}

\subsection{Participants}

Fifteen older adults $(\mathrm{M}=69.8$ and $\mathrm{SD}=7.62)$ and 15 younger adults $(\mathrm{M}=19.2$ and $\mathrm{SD}=1.03)$ participated in this study. All participants were healthy with no known musculoskeletal injuries or neurological disorders that could impair their ability to maintain balance and had normal or correct to normal vision. Older adults were enrolled in activities at University where this study took place and the younger ones were either undergraduate or graduate students. All of them gave their informed consent prior to participation in the experimental session according to procedures approved by the Institutional Review Board.

\subsection{Procedures}

Participants were asked to maintain upright stance inside a moving room at $1 \mathrm{~m}$ away from the frontal wall and to look at a target attached at this frontal wall. The moving room consisted of three walls and a roof $(2.1 \mathrm{~m}$ long x $2.1 \mathrm{~m}$ wide $\mathrm{x} 2.1 \mathrm{~m}$ height), mounted on wheels so that it could be moved back and forth by a servomotor mechanism while the floor remained motionless. The walls and the roof were white with black stripes painted, creating a pattern of $42 \mathrm{~cm}$ wide vertical white and 22 $\mathrm{cm}$ wide vertical black stripes. A 20-watt fluorescent lamp was attached to the ceiling and used to maintain consistent light condition throughout data collection.

The servomotor mechanism consisted of a controller (Compumotor, Model APEX 6151), a controlled stepper motor (Compumotor, Model N0992GR0NMSN), and an electrical cylinder (Compumotor, Model EC3-X3xxN10004a-Ms1-MT1M), which connected the servomotor to the moving room's structure. Specialized software (Compumotor, Motion Architect for Windows) controlled the servomotor mechanism, moving the room continuously away from and toward the participant (anterior/posterior direction). The moving room was oscillated at $0.2 \mathrm{~Hz}$ frequency, with amplitude either of 0.6 $\mathrm{cm}$ and peak velocity of $0.6 \mathrm{~cm} / \mathrm{s}$ or with amplitude of $3.5 \mathrm{~cm}$ and peak velocity of $3.5 \mathrm{~cm} / \mathrm{s}$.

A movement analysis system (OPTOTRAK 3020-3D Motion Measurement System, Northern Digital Inc.) was placed behind the participants. One infrared emitter was placed on the participant's back (at the 8th thoracic vertebra level) and another one on the frontal wall of the moving room. These emitters provided information about the participant's trunk sway and moving room displacement, respectively, in the anterior-posterior (AP), medial-lateral (ML) and vertical directions, with a sampling rate of $100 \mathrm{~Hz}$.

For each participant, 7 trials of 60 seconds apiece were collected. The first three trials were named pre-changing trials and the room was oscillated with a peak velocity of $0.6 \mathrm{~cm} / \mathrm{s}$ and amplitude of $0.5 \mathrm{~cm}$. The fourth trial was named changing trial and the room was oscillated with a peak velocity of $3.5 \mathrm{~cm} / \mathrm{s}$ and amplitude of $3.5 \mathrm{~cm}$. The 
last three trials were named post-changing trials and the room was oscillated again with peak velocity of $0.6 \mathrm{~cm} / \mathrm{s}$ and amplitude of $0.5 \mathrm{~cm}$.

Right after the pre-changing trials, the changing trial, and at the end of the experimental procedures, participants were asked if they had noticed anything different or uncommon during the experimental session. Differently from younger adults that reported that the room had moved after the fourth trial (changing trial), older adults were either not able to discriminate any change in the room's movement or reported that something different/ strange had happened as they stood still.

\subsection{Data Analysis}

As visual stimulus manipulation was in the anterior-posterior (AP) direction, analyses were performed in this direction only and divided in two groups. The first group of analysis examined the magnitude of participants' body sway through the variable mean sway amplitude. Mean sway amplitude was obtained by subtracting a first order polynomial from the signal of each trial. This procedure eliminated any low-frequency body sway changes during the trial that were not related to body oscillation. Then, the mean sway variability was calculated by obtaining the standard deviation of the trunk sway signal. The mean sway amplitude, therefore, corresponded to the variance of the trunk sway, and was used to examine the average performance of the postural control system.

The coupling between visual information and trunk sway was examined through coherence and gain. Coherence measured how strongly body sway was coupled to the visual stimulus, calculated at a frequency of $0.2 \mathrm{~Hz}$. Coherence values close to 1 indicate that the signals demonstrate a strong dependency between them. Conversely, values close to zero indicate that the signals demonstrate a weak or no dependency between them. Gain corresponded to the ratio between the body response amplitude and the visual stimulus amplitude. A gain of one indicates the response amplitude is equal to the stimulus amplitude. To calculate gain, the frequency response function was derived from the trunk sway Fourier Transforms divided by the visual stimulus Fourier Transforms. Gain was computed as the absolute value of the transfer function, at the driving frequency (i.e. $0.2 \mathrm{~Hz}$ ) for each participant.

\subsection{Statistical Analysis}

After testing normality and homogeneity of variance, three analyses of variance (ANOVAs) were performed to verify mean sway amplitude, coherence, and gain between groups and among the three first trials (prechanging), this last factor treated as repeated measures. A multivariate analysis of variance (MANOVA) was performed to verify mean sway amplitude, coherence, and gain between groups in the fourth trial (changing). Finally, three other ANOVAs were performed to verify mean sway amplitude, coherence, and gain between groups and among the three last trials (post-changing), this last factor treated as repeated measures. When appropriate, univariate tests were performed and the $\alpha$-level for all analyses was 0.05 .

\section{RESULTS}

Visual information manipulation, due to the movement of the room, induced corresponding body sway in both older and younger adults. Figure 1 depicts exemplar time series of an older adult in the pre- and in the changing trial. As one can see, body sway was coupled to moving room displacement in both conditions. In addition, MANOVA revealed that, in the changing trial, older adults responded and coupled to the moving room differently from younger adults, Wilks' Lambda $=0.698$, $\mathrm{F}_{3.26}=3.75, \mathrm{p}<0.05$. Following we present the analyses for each variable.

\subsection{Mean Sway Amplitude}

Figure 2 depicts mean sway amplitude across trials for both older and younger adults. Abrupt change in the visual stimulus amplitude and velocity induced larger body sway in older compared to younger adults. ANOVAs revealed no group and trial effects, and no group and trial interaction in the pre-changing trials and in the postchanging trials. However, univariate analysis for the changing trial indicated that body sway was larger in older than in younger adults, $\mathrm{F}_{1.28}=6.25, \mathrm{p}<0.05$.

\subsection{Coherence}

Figure 3 depicts coherence values between the visual stimulus and body sway across trials for both older and younger adults. ANOVAs revealed no group and trial effects, and no group and trial interaction in the prechanging trials and in the post-changing trials. However, univariate analysis for the changing trial indicated that older adults couple to visual information more strongly than younger adults, $\mathrm{F}_{1.28}=4.16, \mathrm{p}=0.05$.

\subsection{Gain}

Figure 4 depicts gain values between the visual stimulus and body sway across trials for both older and younger adults. ANOVAs revealed no group and trial effects, and no group and trial interaction in the prechanging trials, but revealed group effect in the postchanging trials with postural sway of older adults being more induced by visual manipulation compared to 


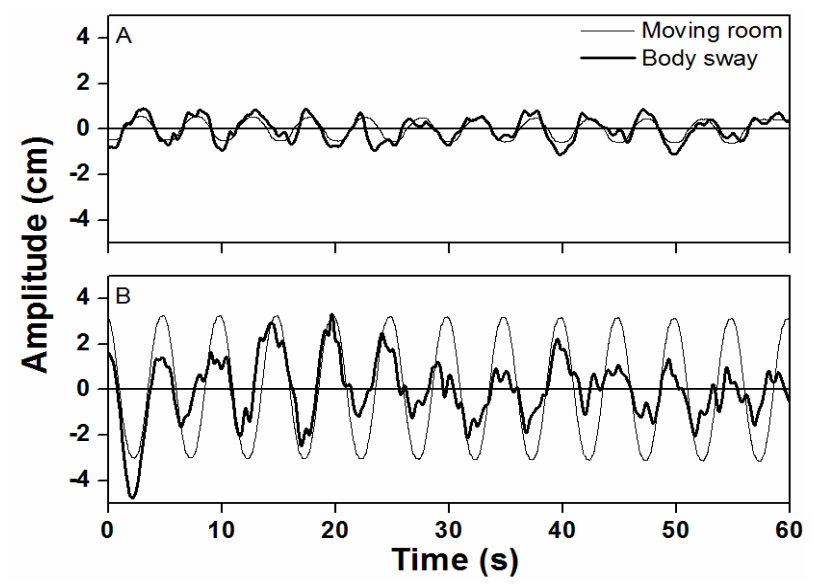

Figure 1. Exemplar time series of body sway of an older adult during a pre-changing trial (top panel) and a changing trial (bottom panel).

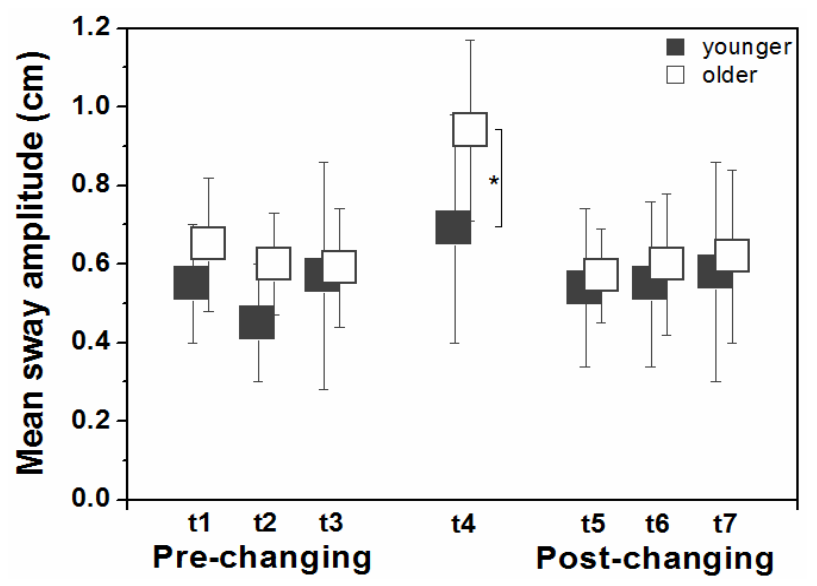

Figure 2. Mean ( \pm standard deviation) values of mean sway amplitude for younger and older adults in the anteriorposterior direction according to each trial (t1, t2, t3, t4, t5, t6,

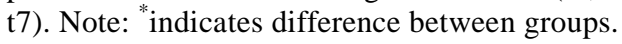

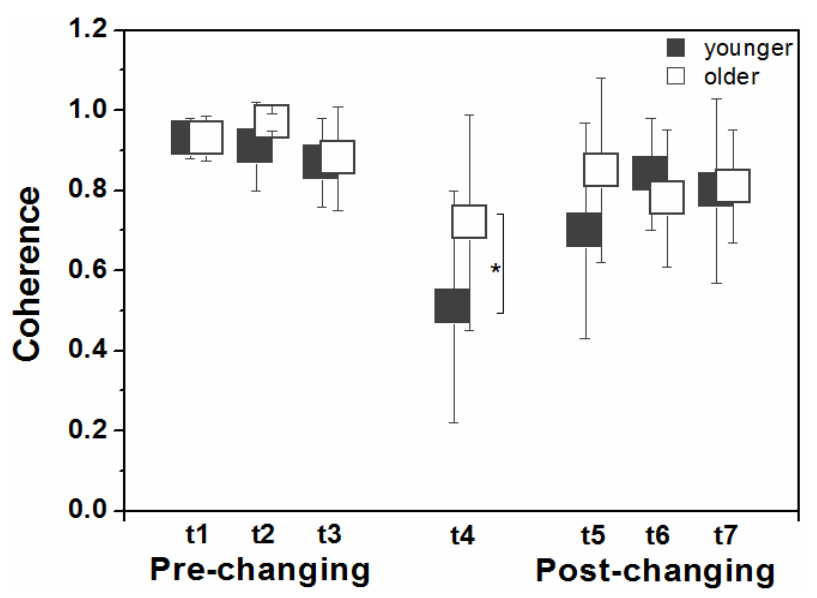

Figure 3. Mean ( \pm standard deviation) values of coherence for younger and older adults in the anterior-posterior direction according to each trial (t1, t2, t3, t4, t5, t6, t7). Note: *indicates difference between groups.

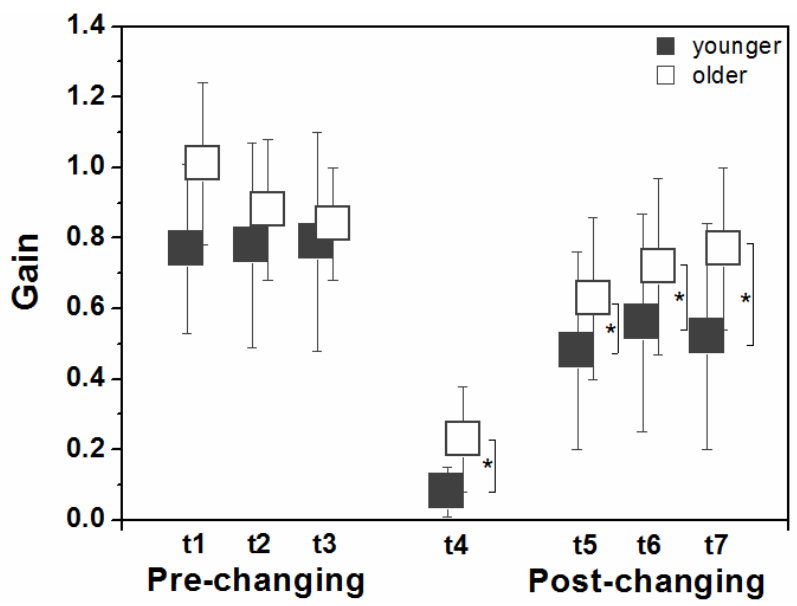

Figure 4. Mean ( \pm standard deviation) values of gain for younger and older adults in the anterior-posterior direction according to each trial $(\mathrm{t} 1, \mathrm{t} 2, \mathrm{t} 3, \mathrm{t} 4, \mathrm{t} 5, \mathrm{t} 6, \mathrm{t} 7)$. Note: *indicates difference between groups.

younger adults, $\mathrm{F}_{1 \cdot 28}=5.56, \mathrm{p}<0.05$. Similar results were observed in the changing trial, $\mathrm{F}_{1.28}=11.86, \mathrm{p}<$ 0.005 , when it was observed a sharp drop in gain values compared to the pre- and post-changing trials, but less accentuated in older than in younger adults.

\section{DISCUSSION}

In the present study, we examined the adaptation due to visual stimulus manipulation over different trials by increasing/decreasing stimulus properties. We observed that older and younger adults adapted to abrupt changes in the visual stimulus, but the magnitude of such adaptation was less accentuated in older than younger adults. More importantly, however, we observed that older adults did not carry over the effects of the visual manipulation from previous to the subsequent expositions. This observation is an indicative that older adults are less adaptable to continuously changing the environmental stimulus.

Adaptation to abrupt visual stimulus alteration in older adults has been observed in previous studies $[17,18]$. Our results corroborate these previous findings. Actually, such a mechanism is quite clever to prevent threatening to the postural orientation and equilibrium which is present in children [9], younger [12] and older adults [18]. It evolves through expositions and experiences in order to the central nervous system to learn how to extract from among multiple sources of sensory cues the most relevant and useful ones for the task and condition at hand.

Despite of being able to adapt to environmental alterations, it has been suggested that older adults differ from younger ones. Some researchers have shown that aging slows the process of sensory reweighting, leading to a longer adaptation period when alterations in sensory cues occur $[17,19,21]$. However, with sufficiently long expo- 
sition to the sensory conditions, older adults can adapt their responses similarly to the younger adults' behavior [18]. Therefore, such difference in the time scale adaptation could explain why older adults are quite more influenced by any dramatic sensory alteration $[18,24,25]$, as it would take longer period to resolve the conflicting sensory situation [17] and carry over such adaptation to the following visual stimulus exposures.

Although healthy older adults show improved stability on repeated trials in which sensory conditions are maintained [e.g., 26], our results demonstrate that older adults use less efficiently previous experiences to modulate subsequent expositions compared to younger adults. Gain values were higher in the post-changing trials for the older than for the younger adults, indicating that differently from older adults, younger ones were able to "learn" that visual manipulation occurred between the pre- and post-changing trials and, therefore, visual influences should be down weighted in order to prevent unnecessary postural responses.

These results suggest that the central nervous system mechanisms and the ability to fuse sensory cues coming from multiple sources seem to be intact in older adults to control the upright stance, as recently suggested [19]. However, the natural aging process definitely affects the calibration of the parameters involved in this process. Older adults not only reduced less postural response due to increased visual amplitude manipulation but also learned less based upon such manipulation compared to younger adults. The ability to predict the effects of an action is a fundamental brain function achieved through continuous update of the internal model [27]. Such updating is based upon the previous experience. But in the case older adults, it might be slightly compromised, as previously suggested [28].

A potential cause preventing a precise internal model updating, leading to a less calibrated response, would be related to peripheral sensory changes that occur due to aging. For instance, postural control is compromised due to peripheral sensory changes in older adults [e.g., 15,29]. Therefore, older adults might use less accurate sensory cues coming from the periphery informing body dynamics and, consequently, not adapt as efficiently as young adults in situations that require a prompt postural response. As a consequence, any sensory manipulation induces larger postural response in older than in younger adults $[14,17,20,30]$ and even other related motor tasks [28]. Because less accurate cues are available to older adults, the central nervous system needs to be exposed longer to use such exposition for future situations, as observed in our results.

\section{CONCLUSION}

In summary, older adults adapt to abrupt changes in the visual stimulus. However, the magnitude of such adaptation was less accentuated than the one observed for younger adults. Subsequent responses are less affected by previous experiences, indicating that older adults are less adaptable to continuously change the environmental stimulus. That might be due to less accurate sensory cues obtained by the sensory systems.

\section{ACKNOWLEDGEMENTS}

We gratefully acknowledge all participants who volunteered for this study.

\section{REFERENCES}

[1] Polastri, P.F., Barela, J.A., Kiemel, T. and Jeka, J.J. (2012) Dynamics of inter-modality re-weighting during human postural control. Experimental Brain Research, 223, 99108. http://dx.doi.org/10.1007/s00221-012-3244-z

[2] Forssberg, H. and Nashner, L.M. (1982) Ontogenetic development of postural control in man: adaptation to altered support and visual conditions during stance. The Journal of Neuroscience, 2, 545-552.

[3] Nashner, L.M., Black, F.O. and Wall, C. (1982) Adaptation to altered support and visual conditions during stance: Patients with vestibular deficits. The Journal of Neuroscience, 2, 536-544.

[4] Peterka, R.J. (2002) Sensorimotor integration in human postural control. Journal of Neurophysiology, 88, 10971118.

[5] Oie, K.S., Kiemel, T. and Jeka, J.J. (2001) Human multisensory fusion of vision and touch: Detecting nonlinearity with small changes in the sensory environment. Neuroscience Letters, 315, 113-116.

[6] Jeka, J.J., Oie, K.S. and Kiemel, T. (2008) Asymmetric adaptation with functional advantage in human sensorimotor control. Experimental Brain Research, 191, 453463. http://dx.doi.org/10.1007/s00221-008-1539-x

[7] Mergner, T., Maurer, C. and Peterka, R.J. (2003) A multisensory posture control model of human upright stance. Progress in Brain Research 142, 189-201. http://dx.doi.org/10.1016/S0079-6123(03)42014-1

[8] Barela, J.A., Toledo, D.R., Ferreira, D.M.A. and Polastri, P.F. (2009) Repesagem e adaptação sensorial no controle postural de adultos. Neurociências 5, 141-149.

[9] Rinaldi, N.M., Polastri, P.F. and Barela, J.A. (2009) Agerelated changes in postural control sensory reweighting. Neuroscience Letters, 467, 225-229. http://dx.doi.org/10.1016/j.neulet.2009.10.042

[10] Kiemel, T., Oie, K.S. and Jeka, J.J. (2002) Multisensory fusion and the stochastic structure of postural sway. Biological Cybernetics, 87, 262-277. http://dx.doi.org/10.1007/s00422-002-0333-2

[11] van der Kooij, H., Jacobs, R., Koopman, B. and van der Helm, F. (2001) An adaptive model of sensory integration in a dynamic environment applied to human stance control. Biological Cybernetics, 84, 103-115. 
http://dx.doi.org/10.1007/s004220000196

[12] Carver, S., Kiemel, T. and Jeka, J.J. (2006) Modeling the dynamics of sensory reweighting. Biological Cybernetics, 95, 123-134. http://dx.doi.org/10.1007/s00422-006-0069-5

[13] Berger, L., Chuzel, M., Buisson, G. and Rougier, P. (2005) Undisturbed upright stance control in the elderly: Part 1. Age-related changes in undisturbed upright stance control. Journal of Motor Behavior, 37, 348-358. http://dx.doi.org/10.3200/JMBR.37.5.348-358

[14] Prioli, A.C., Cardozo, A.S., de Freitas Junior, P.B. and Barela, J.A. (2006) Task demand effects on postural control in older adults. Human Movement Science, 25, 435446. http://dx.doi.org/10.1016/j.humov.2006.03.003

[15] Sturnieks, D.L., St. George, R. and Lord, S.R. (2008) Balance disorders in the elderly. Neurophysiologie Clinique = Clinical Neurophysiology, 38, 467-478. http://dx.doi.org/10.1016/j.neucli.2008.09.001

[16] Horak, F.B. (2006) Postural orientation and equilibrium: what do we need to know about neural control of balance to prevent falls? Age and Ageing, 35, ii7-ii11. http://dx.doi.org/10.1093/ageing/afl077

[17] Prioli, A.C., Freitas Junior, P.B. and Barela, J.A. (2005) Physical activity and postural control in the elderly: Coupling between visual information and body sway. Gerontology, 51, 145-148. http://dx.doi.org/10.1159/000083984

[18] Jeka, J.J., Allison, L.K. and Kiemel, T. (2010) The dynamics of visual reweighting in healthy and fall-prone older adults. Journal of Motor Behavior, 42, 197-208. http://dx.doi.org/10.1080/00222895.2010.481693

[19] Allison, L.K., Kiemel, T. and Jeka, J.J. (2006) Multisensory reweighting of vision and touch is intact in healthy and fall-prone older adults. Experimental Brain Research, 175, 342-352. http://dx.doi.org/10.1007/s00221-006-0559-7

[20] Wade, M.G., Lindquist, R., Taylor, J.R. and Treat-Jacobson, D. (1995) Optical flow, spatial orientation and the control of posture in the elderly. The Journals of Gerontology, 50B, P51-P58. http://dx.doi.org/10.1093/geronb/50B.1.P51

[21] Teasdale, N. and Simoneau, M. (2001) Attentional demands for postural control: The effects of aging and sensory reintegration. Gait and Posture, 14, 203-210.
[22] Lord, S.R., Tiedemann, A., Chapman, k., Munro, B., Murray, S.M. and Sherrington, C. (2005) The effects of an individualized fall prevention program on fall risk and falls in older people: A randomized, controlled trial. Journal of American Geriatrics Society, 53, 1296-1304. http://dx.doi.org/10.1111/j.1532-5415.2005.53425.x

[23] Anson, E. and Jeka, J.J. (2010) Sensory reweighting: a rehabilitative mechanism? In: C. L. Armstrong and L. Morrow, Eds., Handbook of Medical Neuropsychology, Springer Science, Amsterdam, pp. 519-529. http://dx.doi.org/10.1007/978-1-4419-1364-7_29

[24] Wade, M.G. and Jones, G. (1997) The role of vision and spatial orientation in the maintenance of posture. Physical Therapy, 77, 619-628.

[25] Eikema, D.J., Hatzitaki, V., Tzovaras, D. and Papaxanthis, C. (2012) Age-dependent modulation of sensory reweighting for controlling posture in a dynamic virtual environment. Age, 34, 1381-1392. http://dx.doi.org/10.1007/s11357-011-9310-9

[26] Woollacott, M.H., Shumway-Cook, A. and Nashner, L.M. (1986) Aging and posture control changes in sensory organization and muscular coordination. International Journal of Aging and Human Development, 23, 97-114. http://dx.doi.org/10.2190/VXN3-N3RT-54JB-X16X

[27] Wolpert, D.M., Ghahramani, Z. and Jordan, M.I. (1995) An internal model for sensorimotor integration. Science, 269, 1880-1882. http://dx.doi.org/10.1126/science.7569931

[28] Lafargue, G., Noel, M. and Luyat, M. (2013) In the elderly, failure to update internal models leads to over-optimistic predictions about upcoming actions. PLoS One, $\mathbf{8}$, Article ID: e51218. http://dx.doi.org/10.1371/journal.pone.0051218

[29] Baccini, M., Rinaldi, L.A., Federighi, G., Vannucchi, L., Paci, M. and Masotti, G. (2007) Effectiveness of fingertip light contact in reducing postural sway in older people. Age and Ageing, 36, 30-35. http://dx.doi.org/10.1093/ageing/afl072

[30] Toledo, D.R. and Barela, J.A. (2010) Sensory and motor differences between young and older adults: Somatosensory contribution to postural control. Revista Brasileira de Fisioterapia, 14, 267-275. 